\title{
Impact of Electronic Fluctuations and Their Description on the Exciton Dynamics in the Light-Harvesting Complex PE545
}

Mortaza Aghtar, ${ }^{\dagger, \S}$ Ulrich Kleinekathöfer, ${ }^{*, \dagger}$ Carles Curutchet, ${ }^{\ddagger}$ and Benedetta Mennucci

$\dagger$ Department of Physics and Earth Sciences, Jacobs University Bremen, Campus Ring 1, 28759 Bremen, Germany

$\ddagger$ Departament de Farmàcia $i$ Tecnologia Farmacèutica $i$ Fisicoquímica and Institut de Biomedicina (IBUB), Facultat de Farmàcia, Universitat de Barcelona, Av. Joan XXIII s/n, 08028 Barcelona, Spain

IDipartimento di Chimica e Chimica Industriale, University of Pisa, Via G. Moruzzi 13, I-56124 Pisa, Italy

$\S$ Present address: Institut für Theoretische Physik, Universität Ulm, Albert-Einstein-Allee 11, 89073 Ulm, Germany

E-mail: u.kleinekathoefer@jacobs-university.de 


\begin{abstract}
Temperature-dependent fluctuations of both site energies and electronic couplings are known to affect the excitation energy transfer in light-harvesting complexes. Environment effects on such fluctuations as well as possible spatial correlations among them are here investigated in the PE545 complex from cryptophyte algae using ensembleaveraged wave packet dynamics to extract the exciton dynamics. This strategy directly uses the time-dependent fluctuations of the system Hamiltonian as described by quantum mechanics/molecular mechanics calculations performed along a classical MD trajectory. Neither the fluctuations in the couplings nor spatial correlations including cross-correlations between site energies and couplings are found to be important in the exciton dynamics of the complex. This finding does not change if a polarizable embedding is used instead of its electrostatic counterpart. The impact of variations in spectral densities and screening of excitonic couplings based on the electrostatic and polarizable embeddings are discussed as well.
\end{abstract}




\section{Introduction}

The light harvesting (LH) function of photosynthetic organisms involves a very sophisticated and efficient molecular machinery, through which light is absorbed by pigment-protein complexes and subsequently the excitation energy is transferred to reaction centers where charge separation takes place. ${ }^{1}$ If one aims at modeling such a function, all the components of the system (the pigments, the protein and the surrounding environment) should be included in a dynamic model. Moreover, quantum effects need to be incorporated if the molecular details of excitation energy and charge transfers are pursued but full quantum treatments are only possible for parts of the photosynthetic complex and/or for individual structures. ${ }^{2,3}$ Since biological complexes are rather flexible, however, studies of individual structures have their limitations.

In the present study we investigate the role of structural fluctuations in energy transfer dynamics using an ensemble averaged wave packet approach. This strategy directly uses the time-dependent fluctuations of the system Hamiltonian as described by quantum mechanics/molecular mechanics (QM/MM) calculations performed along a classical molecular dynamics (MD) trajectory. ${ }^{4-6}$ This approach differentiates from the common density matrix approaches where the information regarding time-dependent fluctuations is encapsulated in the so-called spectral density of electron-vibrational coupling. The ensemble averaged wave packet approach thus allows to naturally account for possible correlations involving the fluctuations of site energies and electronic couplings, which describe the system Hamiltonian, or to include local differences in electron-vibrational couplings among individual pigments due to different local environments. Strong correlations between protein-induced fluctuations in the site energies ${ }^{7-9}$ or cross-correlations between site energies and couplings ${ }^{10,11}$ have been suggested earlier as the source of the experimentally observed coherence beatings.

The study focuses on the phycoerythrin 545 (PE545) antenna of Rhodomonas sp. CS24 marine algae. ${ }^{12,13}$ The PE545 complex is present in cryptophyte algae and can be found in both marine and fresh water. The PE545 complex was purified for the first time in $1987^{14}$ and 


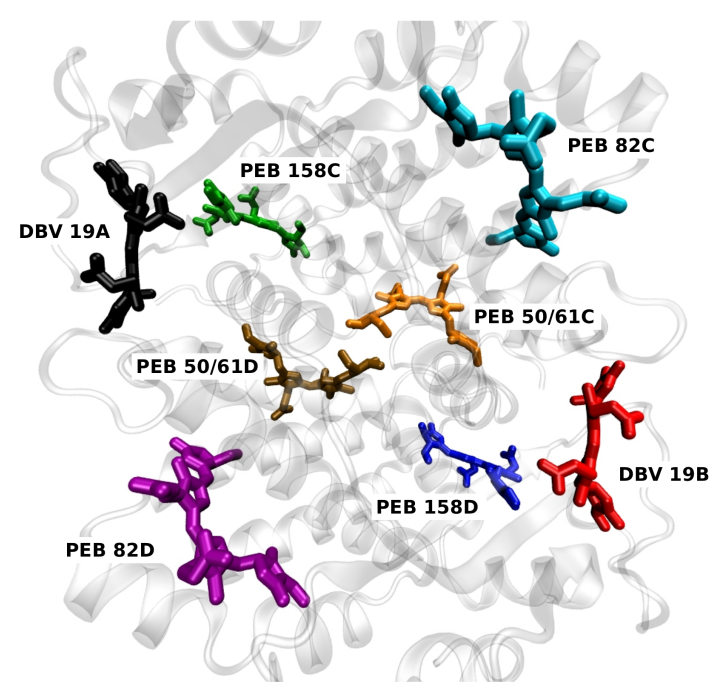

Figure 1: Structure of the PE545 antenna system with the protein scaffold shown in grey cartoon representation and the eight pigments in different colors. The figure was rendered using VMD. ${ }^{22}$

has been structurally resolved to $1.63 \AA$ and $0.97 \AA$ resolutions. ${ }^{15,16}$ These structures allow for a molecular level analysis of the complex. The protein consists of four subunits denoted A, B, C, and D (see Fig. 1) arranged as a $\alpha_{1} \alpha_{2} \beta \beta$ heterodimer. The $\alpha$ subunits each contain a dihydrobiliverdin, DBV, denoted as $\alpha 19$ while three phycoerythrobilins (PEBs) $\beta 50 / \beta 61$, $\beta 82$, and $\beta 158$ are embedded in each $\beta$ subunit. The spectroscopic and photophysical properties of the PE545 have been investigated in several experimental studies. ${ }^{12,13,16-21}$

On the theoretical side, quite a few simulations with a focus on the PE545 complex have been performed. In these studies the parameters have either been extracted mainly from experimental data ${ }^{19,23}$ or from structure-based calculations. ${ }^{5,21,24-28}$ Interestingly, these studies have shown that the rather flexible nature of bilin pigments leads to some special characteristics of the PE545 complex compared to antenna proteins based on (bacterio)chlorophylls. Namely, it has been found that the tuning of the site-energies is performed mainly by constraining the conformation of the pigments in the protein scaffold, that most of the features found in the spectral density arise from intramolecular vibrations of the pigments, and finally that significant cross-correlations in fluctuations of site energies and electronic couplings are present. The aim of the present study is to investigate in detail the impact of environmental 
fluctuations on the exciton dynamics. This analysis is combined with a comparison among different theoretical approaches used to derive the time-dependent Hamiltonian. Aspects being looked at are the effect of environmental polarization on the fluctuations of energy gaps and excitonic couplings as well as possible spatial correlations among the fluctuations of energies and couplings. In order to rationalize the impact of environment polarization in the quantum dynamics, we also compute the spectral densities encoding the couplings between the relevant electronic excited states and the environmental degrees of freedom.

\section{Methods}

Closely following our previously described procedure, ${ }^{4,6,26,27,29,30}$ we first determine a classical MD trajectory of the PE545 complex $\left(\mathrm{PDB} 1 \mathrm{XG} 0^{16}\right)$ in water. We employed the AMBER parm99SB ${ }^{31,32}$ force field for the protein together with the TIP3P water model; ${ }^{33}$ the force fields for the bilins were constructed using GAFF. ${ }^{34}$ In order to calculate electrostatic forces, the values for the bilin partial charges were taken from Ref. [24]. After minimization, the system was equilibrated for $12 \mathrm{~ns}$ at $300 \mathrm{~K}$ and 1 atm using NAMD. ${ }^{35}$ A constant volume trajectory of 720 ps length was then generated in which the atomic coordinates were stored every 2 fs, i.e., a total number of 360,000 snapshots were generated. As described before, ${ }^{36}$ a rather short time step between snapshots is needed to capture accurately the high-frequency regime of spectral densities.

Due to the large number of vertical excitation energies which need to be calculated, we limit ourselves to a semi-empirical approach, i.e., Zerner's intermediate neglect of differential overlap with parameters for spectroscopic properties (ZINDO/S). ${ }^{37-39}$ This technique is well suited for the calculation of vertical excitation energies for $\pi$-conjugated systems when combined with the configuration interaction formalism at the single configuration level (ZINDO/S-CIS). Details of the QM/MM and QM/MMPol implementations to include the environmental effects, based on the ORCA $3 \operatorname{code}^{40}$ and a modified version of the GAUSSIAN 
09 code ${ }^{41}$ are included in the next Section. The QM/MM calculations adopted the point charges used in the MD simulation to describe the environment, whereas the QM/MMPol calculations use a polarizable force field based on point charges and isotropic polarizabilities derived from DFT calculations performed on the residues of PE545 and on water using the LoProp approach, ${ }^{42}$ as described in Ref. [24]. In addition, the polarizability of all MM atoms beyond $10 \AA$ from any QM atom was turned off in order to alleviate the computational requirements. While this choice has a small impact on site energies, ${ }^{24}$ it introduces non-negligible errors in the calculation of screening effects in the electronic couplings. For this reason, for each pigment pair we rescaled the corresponding couplings along the MD trajectory by the ratio of the corresponding average screening factor obtained in previous CIS/6-31G MMPol calculations ${ }^{24}$ with a polarization cutoff of $18 \AA$ and the average screening factors obtained from the present calculations.

The exciton dynamics are performed using the ensemble averaged wave packet approach as used earlier for this ${ }^{5}$ and similar systems. ${ }^{6}$ In such a scheme, the fluctuating Hamiltonian is employed with the time divided into short time intervals during which the Hamiltonian can be assumed to be constant. This time interval coincides with the MD time step (in the present case, 2 fs). To obtain a proper ensemble average, the calculation of the exciton dynamics was repeated many times with different starting positions along the MD trajectory. Subsequently, the results are averaged to obtain the final exciton dynamics. As it has been shown for smaller systems, ensemble averaged wave packet dynamics and the respective density matrix calculations yield the same results ${ }^{36}$ though, in the general case, there is a problem with reaching the proper equilibrium state for the former method. 


\section{Results}

\subsection{Site energies}

In a first step, we study how different flavours of the QM/MM method impact the determination of the site energies, and more importantly, their fluctuations. Indeed, whereas the energy values can be refined by comparison to experiments and thus can eventually be shifted along the trajectory before simulating the quantum dynamics, the quality of their fluctuations is more difficult to assess.

In our strategy, all simulations start with a classical ground state MD simulation as discussed in the previous section. Subsequently, the vertical excitation energies of the individual pigments are determined. Since a large number of these excitation energies are necessary, one has to resort to approximate methods. Here, we focus on ZINDO semi-empirical calculations in combination with MM descriptions of the environment based either on the electrostatic or the polarizable embedding.

Dissimilar variants of the electrostatic embedding have been implemented in different computational codes and therefore a preliminary test on the effects of this variable has to be performed. In particular, the ORCA code employs an approach often used for QM/MM coupling in semi-empirical methods basically assuming that each partial charge is representing a hydrogen-like atom with the respective charge. ${ }^{43}$ In the Gaussian code, however, the MM charges are added as extra terms directly into the Hamiltonian. ${ }^{21}$ Nonetheless, as it can be seen in Fig. S1, these approaches do actually lead to almost the same results when used on the same piece of MD trajectory. Another computational parameter to be preliminarily checked concerns the size of the active space used in the ZINDO calculations. Although the original parameterization of ZINDO/S-CIS was performed for small active configuration interaction (CI) spaces of around 50 configurations, ${ }^{37-39}$ i.e. roughly the seven highest occupied and seven lowest unoccupied states denoted by $(7 / 7)$, the method was assumed to work accurately for larger active space (AS) as well. Even the developers of the method 
applied quite large AS without changing the parameterization. ${ }^{44}$ For bacteriochlorophylls and similar molecules the accuracy of different AS sizes was previously investigated. ${ }^{45,46}$ As shown in Fig. S1, the only significant effect of changing the size of the active space from $(10,10)$ to the full $\mathrm{AS}$ is a shift by not more than $0.25 \mathrm{eV}$ for all energies along the trajectory with practically no change in the fluctuation pattern which is of major concern in the present study. Thus, an active space of $(10,10)$ has been employed in the rest of this study since all relative energies and thus the exciton dynamics stay unchanged.

In PE545, where the pigments are covalently linked to the protein scaffold, in order to set the QM/MM calculations one also needs to choose the location of the QM/MM boundary. Test calculations in this direction showed that only marginal differences were found in the predicted energies as long as the main $\pi$ systems of the pigments (the pyrrole rings) were not touched.

In addition to the electrostatic embedding, a polarizable embedding based on induced dipoles (MMPol) is used here. The comparison between the two different embeddings is reported in Fig. 2 for a piece of the trajectory generated with the classical MD. As shown in Fig. 2, the inclusion of the polarization of the environment using the MMPol approach does lead to an overall reduction in the energy gaps by roughly $0.2 \mathrm{eV}$. When comparing the time-dependent site energies along the trajectory, it becomes apparent that this shift is not constant in time but changes of the fluctuation pattern are found.

In order to get deeper insights in the differences between calculations performed with a non-polarizable (MM) and a polarizable environment (MMPol), in Fig. 3 we plot the distribution of site energies, also termed distribution of states (DOS). In both cases, the distributions have symmetric Gaussian shapes. A clear shift of the distributions between the two approaches is visible which does depend on the individual pigments. In case of nonpolarizable ZINDO/MM, the distributions corresponding to the PEB and the DBV pigments are separated, while they are overlapping in the case of ZINDO/MMPol. This finding is partially due to the fact that the distributions are broader in the polarizable case due to the 


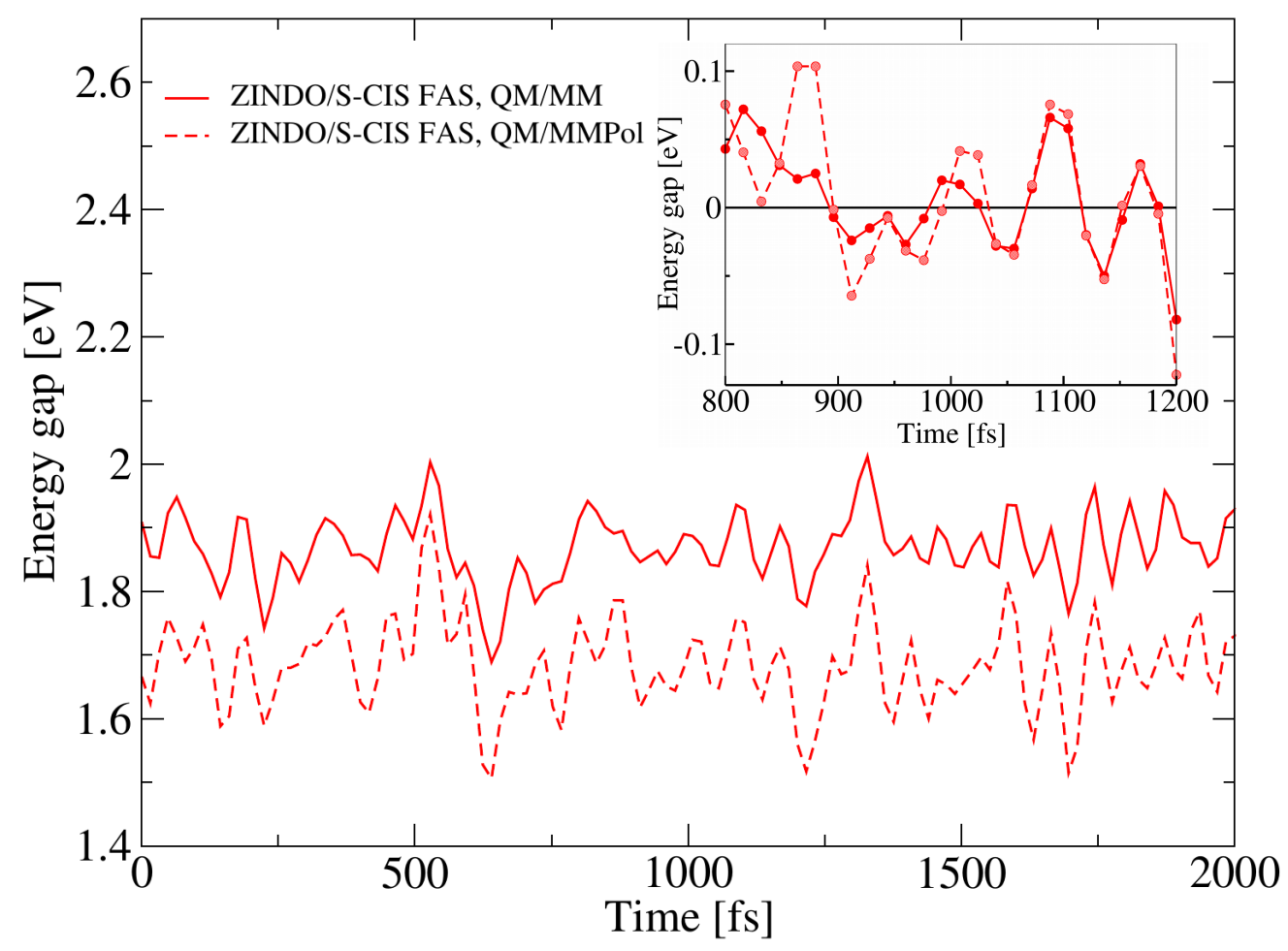

Figure 2: Piece of the energy gap trajectory for pigment DVB19B. Shown are the QM/MM results with full active space (FAS) without $(\mathrm{QM} / \mathrm{MM})$ and with $(\mathrm{QM} / \mathrm{MMPol})$ polarization of the environment. The inset shows a zoomed part of the graph where the average energies have been shifted to the zero value.

larger fluctuations of the site energies. We also note that the variation of the average energies among the pigments predicted from the non-polarizable embedding is larger $(0.49 \mathrm{eV})$ with respect to that found including polarization $(0.38 \mathrm{eV})$ and their ordering is different. All these findings point to a varying effect of polarization on dissimilar local environments.

In order to better understand the impact of polarization in the fluctuations of site energies, we subsequently computed the spectral density of each pigment. This quantity describes the coupling of the excitation in each pigment with the inter and intra-molecular vibrational modes modeled by a bath of harmonic oscillators, and is a key ingredient for density matrixbased calculations of the exciton dynamics. The spectral density can be computed using a half-sided Fourier transform of the site energy autocorrelation function combined with a thermal prefactor. ${ }^{4}$ Here we employed the same procedure used for PE545 in earlier studies. ${ }^{27,28}$ Interestingly, previous work showed that the spectral densities almost do not change if the 


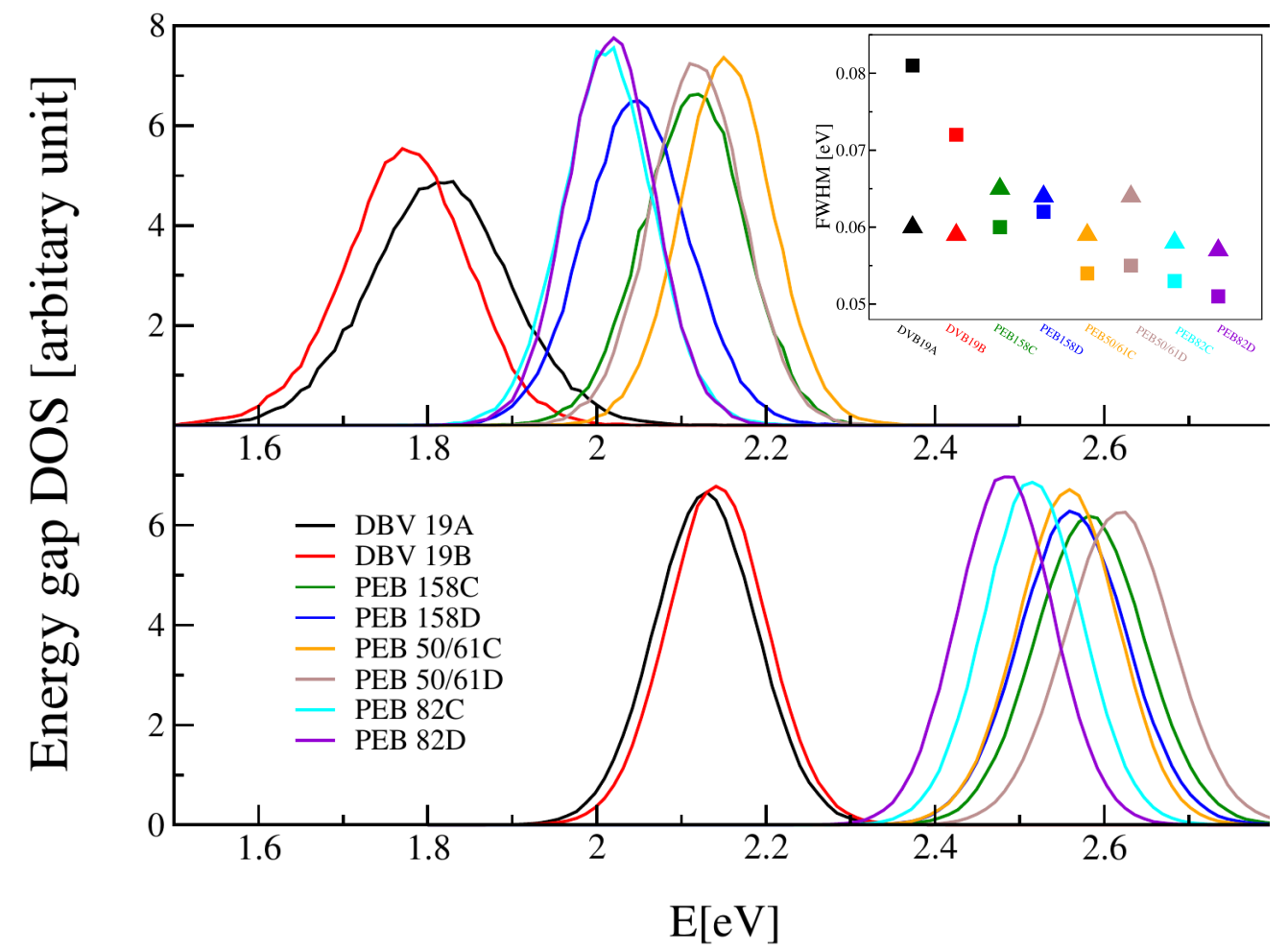

Figure 3: State density of site energies for ZINDO/MMPol (upper plot) and ZINDO/MM (lower plot). The inset shows the full width at half maximum (FWHM) of a Gaussian fit to the distributions of the individual bilins for ZINDO/MMPol (squares) and ZINDO/MM (triangles).

MM environment is kept frozen, indicating that its contribution is negligible or, in other words, that the spectral densities almost exclusively stem from intra-molecular vibrations. ${ }^{28}$ Here, we investigate the impact of polarization in the energy fluctuations by comparing spectral densities obtained using a flexible environment, in which polarization effects are either turned on or off. In particular, we focus on the low-energy part of the spectral densities below $0.1 \mathrm{eV}$, where environmental effects are expected to be larger. The results are reported in Fig. 4 together with the experiment-based average spectral density from Ref. 23

Despite the differences apparent in the fluctuations shown in Fig. 2, the spectral densities shown in Fig. 4 are quite similar indicating the robustness of the method. Especially for the PEB pigments, the similarity of the results is very high already for very low energies. We note that both sets of computations rely on the same MD trajectory: this was not the case for the larger deviations seen previously for the FMO complex. ${ }^{47}$ The polarizable environment 


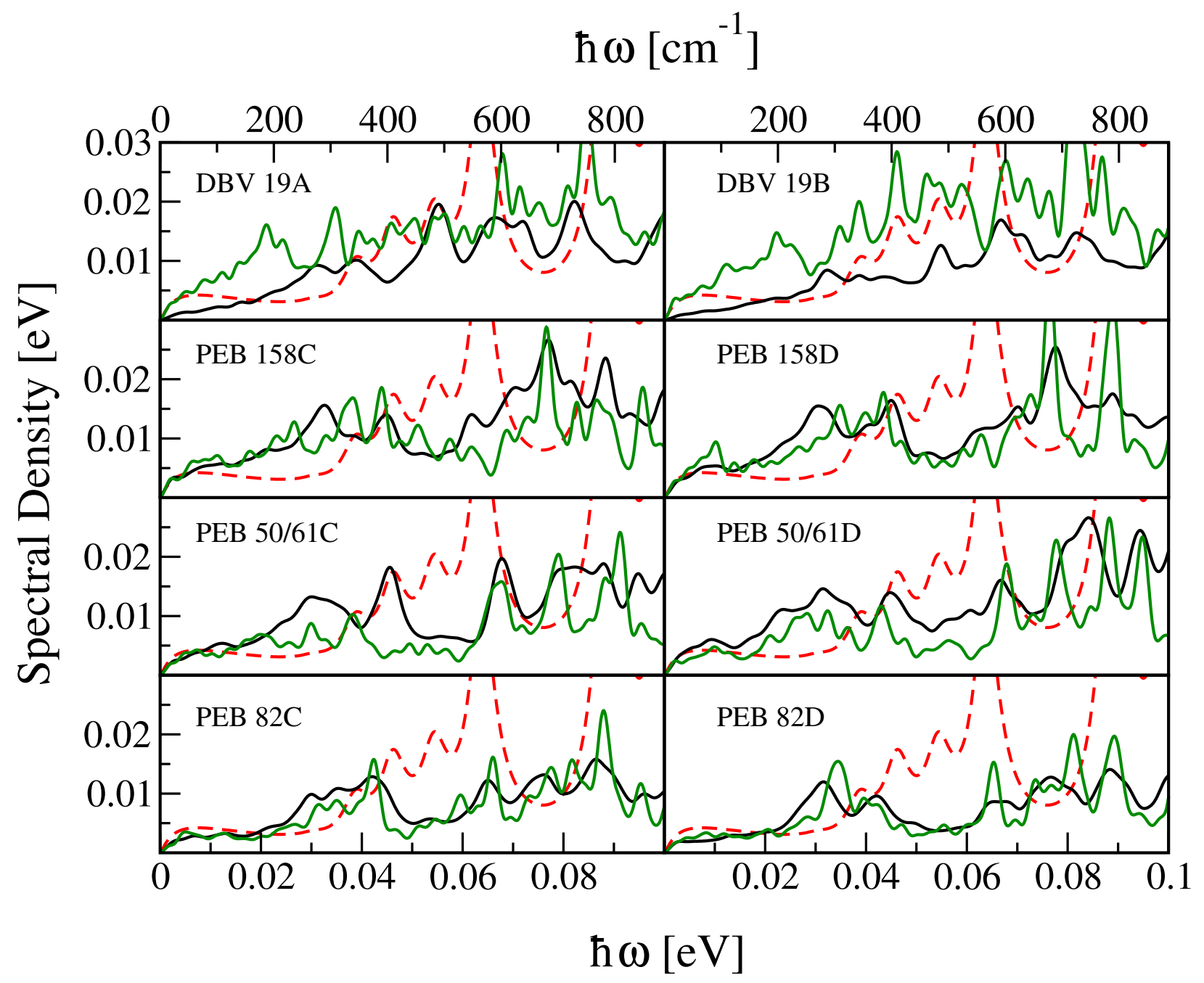

Figure 4: Spectral densities of the 8 bilins calculated with ZINDO/MMPol (solid green) and ZINDO/MM (solid black) compared to the experiment-based average spectral density (dashed red) from Ref. 23.

seems to have a moderate influence on the results, which is maybe not too surprising since the environment in general does not play a major role in the PE545 spectral density. For the DBV bilins the differences are a bit more visible, especially at very low energies, and are larger for DBV 19B than for DBV 19A. Based on experimental spectra, an average spectral density has been constructed ${ }^{23}$ which has a rather similar overall magnitude in the low frequency range, as shown in Fig. 4 and S2, though a strong feature at around $0.065 \mathrm{eV}$ cannot be reproduced by the simulations. 


\subsection{Excitonic couplings}

In order to examine the impact of screening effects and orientations of the pigments, below we introduce three different sets of coupling values, which we later adopt to simulate the quantum dynamics in the complex. One set is based on the point-dipole approximation (PDA) together with the ZINDO transition dipole moments. Environmental effects are included using an exponentially distance-dependent screening factor introduced by Scholes et al. ${ }^{48}$

$$
f\left(R_{m n}\right)=A \exp \left(-\beta R_{m n}\right)+f_{0}
$$

with $A=2.68, \beta=0.271 / \AA$, and $f_{0}=0.54$. The distances between the chromophores $m$ and $n$ are denoted by $R_{m n}$ and are evaluated along the MD trajectory. In the second approach,

the couplings are determined using the MMPol polarizable embedding. ${ }^{24,49}$ This approach automatically includes the effect of the polarizable environment of the chromophores at each snapshot of the MD, thus accounting for their full impact on coupling fluctuations. This scheme includes the explicit atomic details of the environment and its polarizability, and therefore should yield an accurate set of couplings without the need for an additional screening factor. In the third approach we tried to join the simplicity of the PDA with the detailed screening from MMPol calculations. In this way we can dissect the impact of the local details of the environment on the couplings by comparison with the PDA set based on the distance-dependent average screening (PDA/Exp set). Thus, an average screening factor for each pigment pair was extracted from the MMPol simulation and applied to the PDA data (PDA/MMPol set).

In Fig. 5 the distributions of the couplings for the three different sets are shown. Despite a general similarity, some differences are found among the three descriptions. The PDA couplings including the exponential screening seem to be smaller than the MMPol values for most of the pigment pairs. Moreover, the distributions are wider for the MMPol approach. 


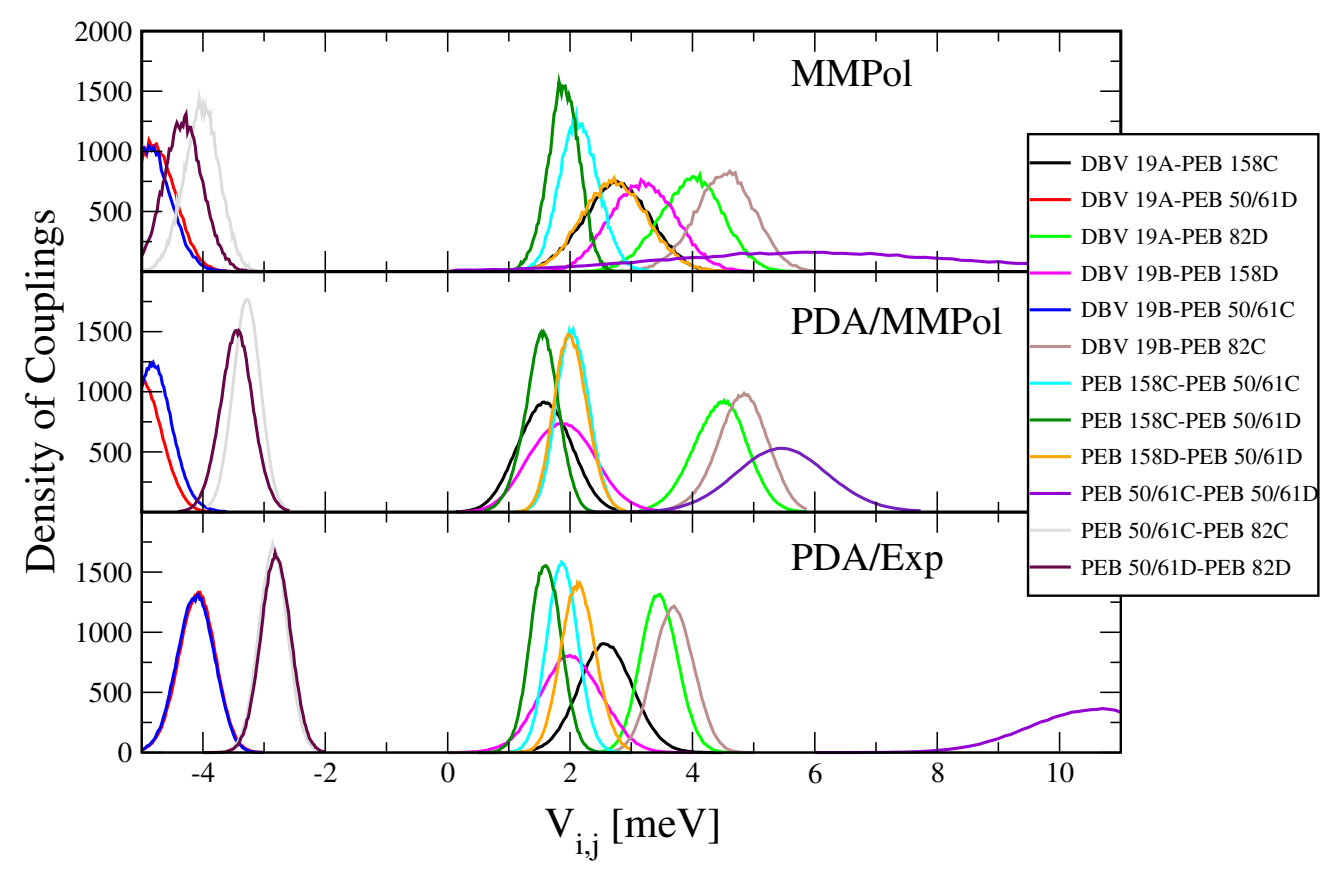

Figure 5: Couplings density of states from MMPol (top), PDA with average MMPol screening (middle), and PDA with exponential screening (bottom). Couplings with absolute average value greater than $1 \mathrm{meV}$ were taken into account.

An exceptional case is the coupling of the central PEB 50/61C-PEB 50/61D pair. In the PDA approach with exponential screening, the average value is above $10 \mathrm{meV}$ while it is around $6 \mathrm{meV}$ for the MMPol scheme. In the later case, however, the width of the distribution is much larger than for all other couplings. Qualitatively, the coupling in this central pigment pair is the largest one. Due to its approximative nature, the point dipole method breaks down at small inter-pigment distances and furthermore is not accurate for certain relative orientations of the respective pigments. ${ }^{50}$

When substituting the unspecific exponential screening to a screening which is pairspecific as extracted from the MMPol calculations for the individual pairs, the largest change again concerns the central PEB pair. Here the fully atomistic nature of the MMPol scheme shows to be clearly superior while the exponential approximation underestimates the screening at this short distances, which again might be strongly dependent on the relative orientation of the interacting pigments. Beyond mutual orientation, however, a previous analysis of screening effects in PE545 indicated a trend toward stronger screening effects at shorter 
interpigment distances due to the heterogeneous nature of the environment, an effect independent of the degree of exposure to the solvent. ${ }^{24}$ This trend probably reflects that, for closely-spaced pigments, the main contributions to the environment-mediated coupling term arise from the local protein environment, whereas for pigments at large separations the water solvent, with a smaller optical dielectric constant compared to the protein, plays a more relevant role.

Thus, the average of the PDA/MMPol coupling for the central PEB pair is now reduced to a value much closer to that of the MMPol approach while the width of the distribution is smaller than that with the exponential screening. Other couplings such as those belonging to the DBV 19A-PEB 82D and the DBV 19B-PEB 82C pairs move to larger values close to those of the MMPol approach. A similar trend can be observed for the larger negative coupling values of, for example, the pairs PEB 50/61C-PEB 82C and PEB 50/61D-PEB 82D.

We can thus conclude that the description of the environmental screening can have a severe influence on the coupling values especially if taking into account the specific environment of each pair. Overall, the similar widths of the distributions suggest that the fluctuations in the couplings described by the three different sets are similar. However, some important differences arise for some pigments, like the central pair, mostly due to the more accurate description of the environment but also because of the better description of the MMPol values based on transition densities compared to the PDA approximation, as shown in Fig. S3 of the Supporting Information.

\subsection{Quantum Dynamics}

Based on the site energy and coupling trajectories, one can construct a time-dependent Hamiltonian for the single-exciton manifold of the PE545 complex. To this end, we do excite an individual pigment and observe how this excitation spreads over all chromophores. Since we employ an ensemble-averaged wave function approach for the quantum dynamics, 
which includes an implicit high-temperature limit, in thermal equilibrium all sites will be equally populated, i.e, the population will be 0.125 in the present system with eight sites. For different initially excited bilins and for three different time-dependent Hamiltonians the exciton population dynamics are shown in Figs. 6 and S4. Three different setups are included: MMPol site energies with MMPol couplings, MMPol site energies with PDA/MMPol couplings, and MM site energies with MMPol couplings. From a comparison of the results for the first and the second Hamiltonian we are able to see the accuracy of the PDA approximation for the present system. For many of the initially excited pigments there is no big difference in the exciton dynamics between the cases with different approximations for the couplings. Only in the case of DBV 19 A the difference is quite obvious and the transfer to PEB 82 D is faster which is consistent with the larger PDA-based coupling as shown in Fig. 5. This is consistent with the larger average coupling value between chromophores DBV 19A and PEB 82D in case of PDA/MMPol compared to MMPol. Comparing the dynamics for MMPol site energies and couplings with those for MM site energies with MMPol couplings we can analyze the effect of the polarization on the site energies since the couplings are determined using the same approach. The differences for these two cases are actually rather small. On additional combination of site energies and couplings is shown in Figs. S7 and S8. Here the MM site energies are combined with the PDA/Exp couplings. The difference to the case MM site energies and MMPol couplings is rather small. For example, the transfer away from DBV 19A is even slightly slower, a finding to be expected from the underlying coupling values.

As can be seen, for some cases, like PEB 158C and PEB 50/61C, the excitation decays from the initially excited pigment obtained for the four different underlying Hamiltonian trajectories are very similar. For other bilins, however, like the two DBVs but also PEB 82D the results based on MMPol site energies and PDA/MMPol couplings differ quite a bit from the three other approaches in that they show a much faster decay rate, suggesting an important breakdown of the PDA approximation. This finding is somewhat surprising since 


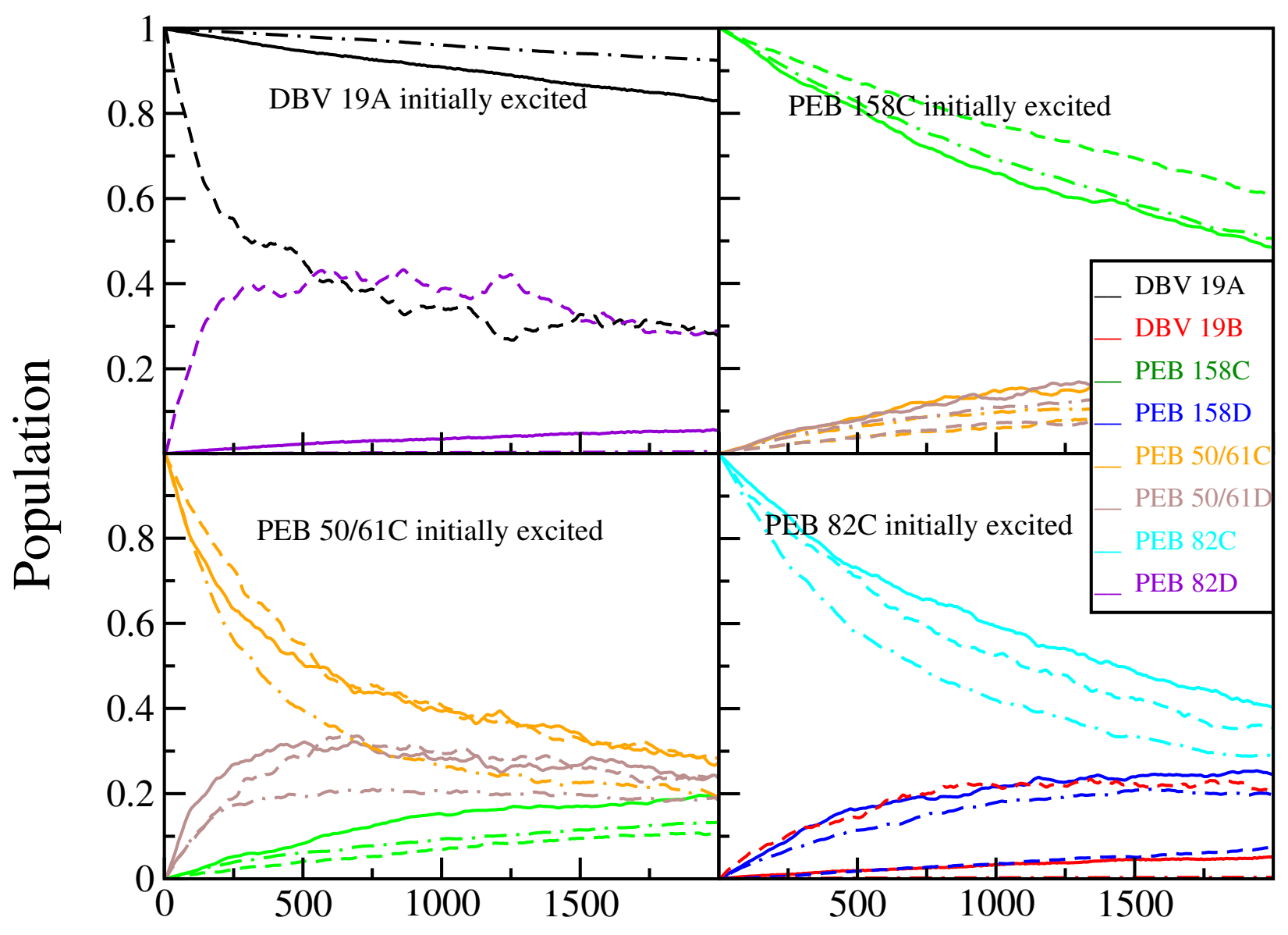

Time $[\mathrm{fs}]$

Figure 6: Excitation dynamics of the bilins with MMPol site energies and couplings (solid line), MMPol site energies and PDA/MMPol couplings (dashed lines), and MM site energies and MMPol couplings (dashed-dotted lines). For each initially excited bilin only the dynamics of bilins to which the population is mostly transfered have been plotted.

the PDA/MMPol couplings are closer to the MMPol couplings than the PDA/Exp couplings. However, in the latter set the MM energies are adopted, which predict a considerably larger energy gap among the DBV 19A/19B pigments and the lowest lying PEBs 82C and 82D, as shown in Fig. 3.

\subsubsection{Fluctuations in couplings and site energies}

In order to analyze the effect of fluctuations in the excitonic couplings and in the site energies separately and independently from shifts of the average site energies, we performed 


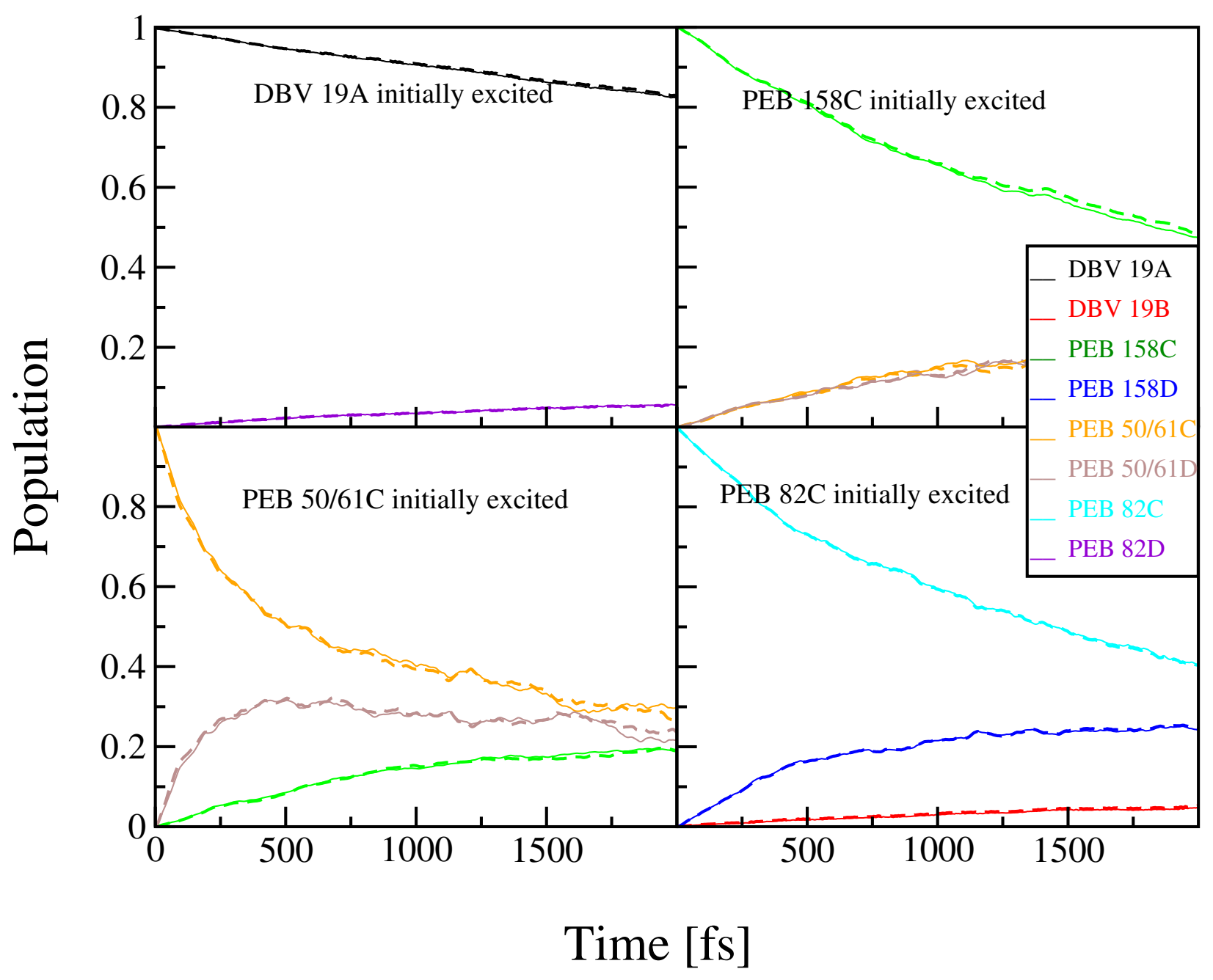

Figure 7: Excitation dynamics of the pigments using MMPol site energies and couplings (dashed lines) compared to the same data while the averaged coupling is used during time (solid thin line).

simulations with the same site energy trajectory from ZINDO/MMPol. In one of the two simulations the couplings are determined using the MMPol approach. In the second simulation, instead, these MMPol coupling trajectories are averaged and only the average values are used as constant coupling in the ensemble-averaged wave packet dynamics. The numerical findings are displayed in Figs. 7 and S5.

As can be seen from these figures, the effect of replacing the time-dependent couplings by their respective average values has only negligible effect on the dynamics. As discussed earlier, most coupling distributions are reasonably narrow and there are no specific enhance- 
ment effects when different couplings have the same value as is the case of resonances for equal site energies. Tests were also performed for PDA couplings with the same qualitative results (data not shown). In similar simulations for charge transfer through $\mathrm{DNA}^{51}$ the same conclusions were drawn, i.e., the transfer dynamics did not change significantly upon averaging of the coupling values.

Next we analyzed the influence of site energies as obtained including or not polarization effects from the environment. As already described, the positions of the DOS for ZINDO/MM and ZINDO/MMPol showed clear differences for the individual pigments (see Fig. 3). Moreover, the widths of these distributions did show some difference albeit not too large. The examination of the corresponding spectral densities shown in Fig. 4, also suggested some differences in the patterns of the fluctuations, especially for the DBVs, which are significantly more coupled to low-frequency vibrations when MM polarization is accounted for. In order to focus the analysis on the different fluctuations described from the two sets of calculations, we shifted all average site energies to the set of energies which have been extracted from an experiment-based refinement of CIS/MMPol calculations. ${ }^{21}$ The site energy trajectories subsequently only differ in their fluctuations. Moreover, the time-averaged MMPol couplings were employed. Figures 8 and S8 show the corresponding excitation dynamics. In this case, differences are visible though they cannot be called significant. The extracted transfer rates would only be shifted in a minor way. Therefore, the inclusion of polarization in the site energy calculations did not lead to a major change when an ensemble averaged wave packet dynamics method is used.

\subsubsection{Influence of correlated fluctuations}

Spatial correlations have shown to significantly influence the charge transport properties in DNA. ${ }^{51,52}$ Moreover, spatial correlations in site energy fluctuations have been suggested as the possible origin for the experimentally observed long-lived quantum coherences in FMO and other systems. ${ }^{7-9}$ Cross-correlations between site energies and couplings were investi- 


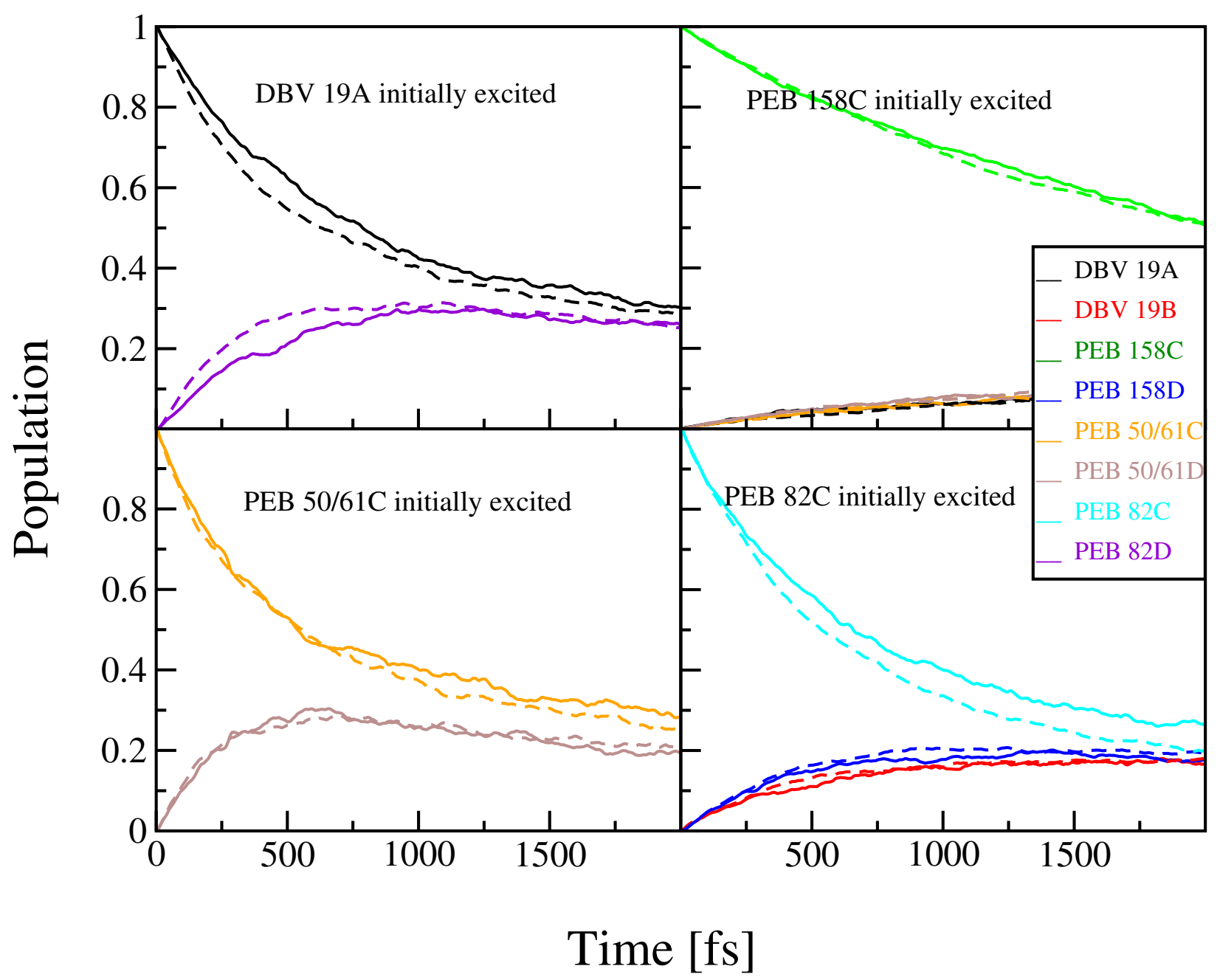

Figure 8: In order to compare the impact of the fluctuations of site energies on excitation dynamics for two methods, MMPol (solid) and MM (dashed), the same couplings set (MMPol) has been used and also the average energies have been fitted to the experiment values

gated as well. ${ }^{10,11}$ In particular, the present authors analyzed the presence of correlations in LH2, ${ }^{29} \mathrm{FMO}^{53}$ and PE545. ${ }^{26}$ For the first two systems, the existence of such correlations was denied though the matter is still under debate. ${ }^{9}$ Concerning the PE545 complex no significant site energy correlations were found though considerable cross-correlations of energies and couplings were observed. ${ }^{26}$

Here we follow a trick introduced by Shim et al. ${ }^{54}$ in which the direct effect of spatial correlation on the exciton dynamics is investigated. In the present context, spatially correlated fluctuations describe a (close) relationship between fluctuations especially of site energies 
of different pigments. These correlations lead to a reduced relative fluctuation prolonging dephasing times. Thus, if one destroys the time dependence between the fluctuations at the different pigments, i.e., if one decorrelates the site energies, the effect of spatial correlations would be destroyed. To this end, we did shift the site energies of each pigment by 2 ps with respect to the pigment with the preceding chromophore number. Thus, for every pigment the site energies were taken from unlike pieces of the trajectories. Even if the fluctuations at different sites, would have been correlated in time, this correlation would be destroyed by this procedure. In Figs. 9 and S9 we compare the result with and without this artificial time shifts in the site energy trajectories. No differences can be observed leading to the conclusion that no correlated fluctuations are present which influence the excitation dynamics, neither site energy correlations nor cross-correlations of energies and couplings. Tests with different time shifts lead to the same conclusions. Moreover, cross-correlations between site energies and couplings should have shown an effect already when replacing the timedependent excitonic coupling by their time-averaged counterparts above. Therefore, we do not have any indication that the experimentally observed long-lived quantum coherences in PE5 $45^{12}$ are connected to spatial correlations or cross-correlations. It is, however, possible that spatial correlations do actually increase the robustness of the exciton transfer rather than its efficiency. ${ }^{55,56}$ This issue cannot be analyzed based on the present simulations.

\section{Conclusions}

We have presented a study on the impact of time-dependent fluctuations of both site energies and excitonic couplings, as well as their theoretical description, on the exciton dynamics in the light-harvesting complex PE545 from cryptophyte algae. Electrostatic and polarizable embeddings have been investigated in combination with the ZINDO level of QM description. Shifted average site energies were found when using the two embeddings as well as different time-dependent fluctuations which however resulted in small differences in the spectral 


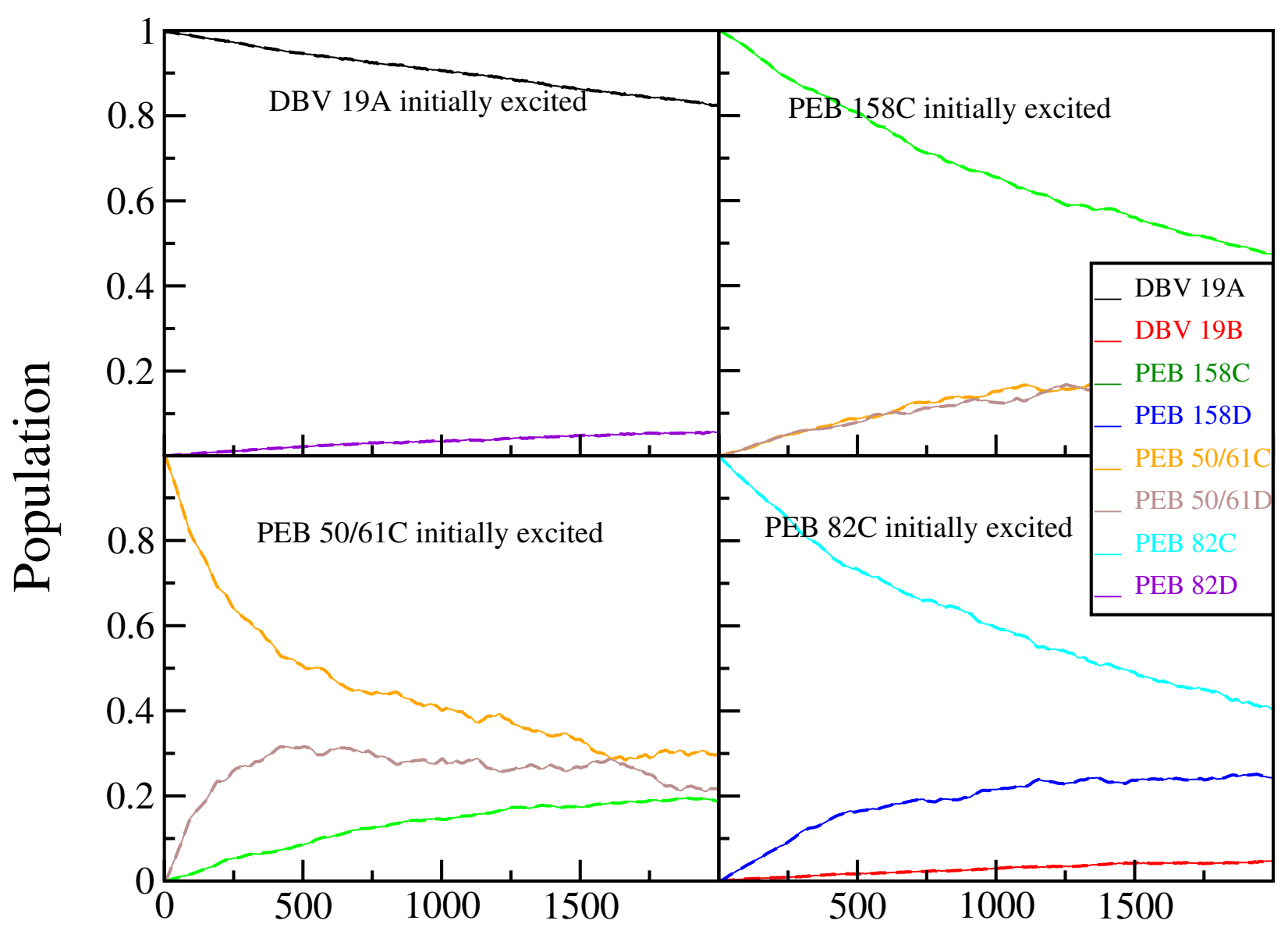

Time $[\mathrm{fs}]$

Figure 9: Thin solid lines correspond to MMPol site energies with averaged PDA/MMPol couplings and the dashed lines correspond to the same data but now the site energies of each pigment has been shifted by 2 ps with respect to the chromophore with the previous pigment number.

densities. The different average site energies were found to have a significant effect on the exciton dynamics whereas replacing the time-dependent couplings by their time-independent averages did not lead to any significant effect. Including environmental polarization in site energy fluctuations, however, lead to small but clearly visible effects. Effects on the exciton dynamics due to the possible correlations of energies and couplings were also investigated but both were found to be not relevant.

We have to stress that the present results are based on a decoupled or sequential approach using a ground state classical MD followed by QM semiempirical calculations of the exci- 
tonic parameters. It is evident that a more accurate MD (for example using a QM/MMPol formulation or excited state trajectories) and a better QM description of the excitonic parameters are necessary to come to a general conclusion about the role of fluctuations and correlations in the formation and relaxation of excitons in the PE545 complex as well as in other light-harvesting complexes.

\section{Supporting Information Available}

The following files are available free of charge. Additional figures are given in the supplementary material.

\section{Acknowledgments}

This work has been supported by grant KL 1299/12-1 of the Deutsche Forschungsgemeinschaft (DFG). C.C. acknowledges support from the Ministerio de Economía y Competitividad (MINECO) of Spain (grants CTQ2012-36195 and RYC2011-08918) and the Agència de Gestió d'Ajuts Universitaris i de Recerca from the Generalitat de Catalunya (GENCAT) (SGR2014-1189).

\section{References}

(1) Blankenship, R. E. Molecular Mechanisms of Photosynthesis; Blackwell Science, 2008.

(2) König, C.; Neugebauer, J. Quantum Chemical Description of Absorption Properties and Excited-state Processes in Photosynthetic Systems. ChemPhysChem 2012, 13, $386-425$.

(3) Curutchet, C.; Mennucci, B. Quantum Chemical Studies of Light Harvesting. Chem. Rev. 2016, (in press, DOI 10.1021/acs.chemrev.5b00700). 
(4) Damjanović, A.; Kosztin, I.; Kleinekathöfer, U.; Schulten, K. Excitons in a Photosynthetic Light-harvesting System: A Combined Molecular Dynamics, Quantum Chemistry and Polaron Model Study. Phys. Rev. E 2002, 65, 031919.

(5) Aghtar, M.; Kleinekathöfer, U. Environmental Coupling and Population Dynamics in the PE545 Light-harvesting Complex. J. Lumin. 2016, 169, 406-409.

(6) Olbrich, C.; Jansen, T. L. C.; Liebers, J.; Aghtar, M.; Strümpfer, J.; Schulten, K.; Knoester, J.; Kleinekathöfer, U. From Atomistic Modeling to Excitation Dynamics and Two-Dimensional Spectra of the FMO Light-Harvesting Complex. J. Phys. Chem. $B$ 2011, 115, 8609-8621.

(7) Engel, G. S.; Calhoun, T. R.; Read, E. L.; Ahn, T. K.; Mancal, T.; Cheng, Y. C.; Blankenship, R. E.; Fleming, G. R. Evidence for Wavelike Energy Transfer Through Quantum Coherence in Photosynthetic Systems. Nature 2007, 446, 782-786.

(8) Cheng, Y. C.; Fleming, G. R. Dynamics of Light Harvesting in Photosynthesis. Annu. Rev. Phys. Chem. 2009, 60, 241-242.

(9) Fokas, A.; Cole, D.; Chin, A. Constrained Geometric Dynamics of the Fenna-MatthewsOlson Complex: The Role of Correlated Motion in Reducing Uncertainty in Excitation Energy Transfer. Photosynth. Res. 2014, 122, 275-292.

(10) Chen, X.; Silbey, R. J. Effect of Correlation of Local Fluctuations on Exciton Coherence. J. Chem. Phys. 2010, 132, 204503.

(11) Huo, P.; Coker, D. F. Influence of Environment Induced Correlated Fluctuations in Electronic Coupling on Coherent Excitation Energy Transfer Dynamics in Model Photosynthetic Systems. J. Chem. Phys. 2012, 136, 115102-17. 
(12) Collini, E.; Wong, C. Y.; Wilk, K. E.; Curmi, P. M. G.; Brumer, P.; Scholes, G. D. Coherently Wired Light-harvesting in Photosynthetic Marine Algae at Ambient Temperature. Nature 2010, 463, 644-647.

(13) Wong, C. Y.; Alvey, R. M.; Turner, D. B.; Wilk, K. E.; Bryant, D. A.; Curmi, P. M. G.; Silbey, R. J.; Scholes, G. D. Electronic Coherence Lineshapes Reveal Hidden Excitonic Correlations in Photosynthetic Light Harvesting. Nat. Chem. 2012, 4, 396-404.

(14) Martin, C. D.; Hiller, R. G. Subunits and Chromophores of a Type I Phycoerythrin from a Chroomonas Sp. (Cryptophyceae). BBA-Gen. Subjects 1987, 923, 88-97.

(15) Wilk, K. E.; Harrop, S. J.; Jankova, L.; Edler, D.; Keenan, G.; Sharples, F.; Hiller, R. G.; Curmi, P. M. G. Evolution of a Light-harvesting Protein by Addition of New Subunits and Rearrangement of Conserved Elements: Crystal Structure of a Cryptophyte Phycoerythrin at 1.63 A Resolution. Proc. Natl. Acad. Sci. USA 1999, 96, 8901-8906.

(16) Doust, A. B.; Marai, C. N.; Harrop, S. J.; Wilk, K. E.; Curmi, P. M.; Scholes, G. D. Developing a Structure-function Model for the Cryptophyte Phycoerythrin 545 using Ultrahigh Resolution Crystallography and Ultrafast Laser Spectroscopy. J. Mol. Biol. 2004, 344, 135-143.

(17) Doust, A. B.; van Stokkum, I. H. M.; Larsen, D. S.; Wilk, K. E.; Curmi, P. M. G.; van Grondelle, R.; Scholes, G. D. Mediation of Ultrafast Light-harvesting by a Central Dimer in Phycoerythrin 545 studied by Transient Absorption and Global Analysis. J. Phys. Chem. B 2005, 109, 14219-14226.

(18) Doust, A. B.; Wilk, K. E.; Curmi, P. M.; Scholes, G. D. The Photophysics of Cryptophyte Light-harvesting. J. Photochem. Photobiol. A: Chem. 2006, 184, 1-17. 
(19) Novoderezhkin, V. I.; Doust, A. B.; Curutchet, C.; Scholes, G. D.; van Grondelle, R. Excitation Dynamics in Phycoerythrin 545: Modeling of Steady-state Spectra and Transient Absorption with Modified Redfield Theory. Biophys. J. 2010, 99, 344-352.

(20) Marin, A.; Doust, A. B.; Scholes, G. D.; Wilk, K. E.; Curmi, P. M. G.; van Stokkum, I. H. M.; van Grondelle, R. Flow of Excitation Energy in the Cryptophyte Lightharvesting Antenna Phycocyanin 645. Biophys. J. 2011, 101, 1004-1013.

(21) Curutchet, C.; Novoderezhkin, V. I.; Kongsted, J.; Munoz Losa, A.; van Grondelle, R.; Scholes, G. D.; Mennucci, B. Energy Flow in the Cryptophyte PE545 Antenna Is Directed by Bilin Pigment Conformation. J. Phys. Chem. B 2013, 117, 4263-4273.

(22) Humphrey, W. F.; Dalke, A.; Schulten, K. VMD - Visual Molecular Dynamics. J. Mol. Graph. 1996, 14, 33-38.

(23) Kolli, A.; O’Reilly, E. J.; Scholes, G. D.; Olaya Castro, A. The Fundamental Role of Quantized Vibrations in Coherent Light Harvesting by Cryptophyte Algae. J. Chem. Phys. 2012, 137, 174109-15.

(24) Curutchet, C.; Kongsted, J.; Munoz Losa, A.; Hossein Nejad, H.; Scholes, G. D.; Mennucci, B. Photosynthetic Light-harvesting Is Tuned by the Heterogeneous Polarizable Environment of the Protein. J. Am. Chem. Soc. 2011, 133, 3078-3084.

(25) Hossein-Nejad, H.; Curutchet, C.; Kubica, A.; Scholes, G. D. Delocalization-Enhanced Long-Range Energy Transfer between Cryptophyte Algae PE545 Antenna Proteins. J. Phys. Chem. B 2011, 115, 5243-5243.

(26) Viani, L.; Curutchet, C.; Mennucci, B. Spatial and Electronic Correlations in the PE545 Light-Harvesting Complex. J. Phys. Chem. Lett. 2013, 4, 372-377.

(27) Viani, L.; Corbella, M.; Curutchet, C.; O'Reilly, E. J.; Olaya Castro, A.; Mennucci, B. 
Molecular Basis of the Exciton-phonon Interactions in the PE545 Light-harvesting Complex. Phys. Chem. Chem. Phys. 2014, 16, 16302-16311.

(28) Aghtar, M.; Strümpfer, J.; Olbrich, C.; Schulten, K.; Kleinekathöfer, U. Different Types of Vibrations Interacting with Electronic Excitations in Phycoerythrin 545 and FennaMatthews-Olson Antenna Systems. J. Phys. Chem. Lett. 2014, 5, 3131-3137.

(29) Olbrich, C.; Kleinekathöfer, U. Time-dependent Atomistic View on the Electronic Relaxation in Light-Harvesting System II. J. Phys. Chem. B 2010, 114, 12427-12437.

(30) Olbrich, C.; Kleinekathöfer, U. From Atomistic Modeling to Electronic Properties of Light-Harvesting Systems. Quantum Efficiency in Complex Systems, Part II: from Molecular Aggregates to Organic Solar Cells. 2011; pp 83-114.

(31) Wang, J.; Cieplak, P.; Kollman, P. A. How Well Does a Restrained Electrostatic Potential (RESP) Model Perform in Calculating Conformational Energies of Organic and Biological Molecules? J. Comput. Chem. 2000, 21, 1049-1074.

(32) Hornak, V.; Abel, R.; Okur, A.; Strockbine, B.; Roitberg, A.; Simmerling, C. Comparison of Multiple Amber Force Fields and Development of Improved Protein Backbone Parameters. Proteins: Struct., Funct., Bioinf. 2006, 65, 712-725.

(33) Jorgensen, W. L.; Chandrasekhar, J.; Madura, J. D.; Impey, R. W.; Klein, M. L. Comparison of Simple Potential Functions for Simulating Liquid Water. J. Chem. Phys. 1983, 79, 926-935.

(34) Wang, J.; Wolf, R. M.; Caldwell, J. W.; Kollman, P. A.; Case, D. A. Development and Testing of a General Amber Force Field. J. Comput. Chem. 2004, 25, 1157-1174.

(35) Phillips, J. C.; Braun, R.; Wang, W.; Gumbart, J.; Tajkhorshid, E.; Villa, E.; Chipot, C.; Skeel, R. D.; Kale, L.; Schulten, K. Scalable Molecular Dynamics with NAMD. J. Comput. Chem. 2005, 26, 1781-1802. 
(36) Aghtar, M.; Liebers, J.; Strümpfer, J.; Schulten, K.; Kleinekathöfer, U. Juxtaposing Density Matrix and Classical Path-based Wave Packet Dynamics. J. Chem. Phys. 2012, 136, 214101.

(37) Ridley, J.; Zerner, M. C. An Intermediate Neglect of Differential Overlap Technique for Spectroscopy: Pyrrole and the Azines. Theor. Chim. Acta 1973, 32, 111-134.

(38) Zerner, M. C.; Loew, G. H.; Kirchner, R. F.; Mueller Westerhoff, U. T. An Intermediate Neglect of Differential Overlap Technique for Spectroscopy of Transition-metal Complexes. Ferrocene. J. Am. Chem. Soc. 1980, 102, 589-599.

(39) Li, J.; Williams, B.; Cramer, C. J.; Truhlar, D. G. A Class IV Charge Model for Molecular Excited States. J. Chem. Phys. 1999, 110, 724-733.

(40) Petrenko, T.; Neese, F. Analysis and Prediction of Absorption Band Shapes, Fluorescence Band Shapes, Resonance Raman Intensities, and Excitation Profiles Using the Time-dependent Theory of Electronic Spectroscopy. J. Chem. Phys. 2007, 127, 164319.

(41) Frisch, M. J.; Trucks, G. W.; Schlegel, H. B.; Scuseria, G. E.; Robb, M. A.; Cheeseman, J. R.; Scalmani, G.; Barone, V.; Mennucci, B.; Petersson, G. A. et al. Gaussian 09 Revision A.02. 2009; Gaussian Inc. Wallingford CT 2009.

(42) Gagliardi, L.; Lindh, R.; Karlstrom, G. Local properties of quantum chemical systems: The LoProp approach. J. Chem. Phys. 2004, 121, 4494-4500.

(43) Alberti, S. F.; Echave, J. Theoretical Study of the Solvatochromism of a Merocyanine Dye. Chem. Phys. 1997, 223, 183-194.

(44) Cory, M. G.; Hirose, H.; Zerner, M. C. Calculated Structures and Electronic Absorption Spectroscopy for Magnesium Phthalocyanine and Its Anion Radical. Inorg. Chem. 1995, 34, 2969-2979. 
(45) Linnanto, J.; Korppi Tommola, J. Spectroscopic Properties of Mg-chlorin, Mg-porphin and Chlorophylls A, B, $\mathrm{C}_{1}, \mathrm{C}_{2}, \mathrm{C}_{3}$ and D Studied by Semi-empirical and Ab Initio MO/CI Methods. Phys. Chem. Chem. Phys. 2000, 2, 4962-4970.

(46) Linnanto, J.; Korppi Tommola, J. Quantum Chemical Simulation of Excited States of Chlorophylls, Bacteriochlorophylls and Their Complexes. Phys. Chem. Chem. Phys. 2006, 8, 663-667.

(47) Chandrasekaran, S.; Aghtar, M.; Valleau, S.; Aspuru-Guzik, A.; Kleinekathöfer, U. Influence of Force Fields and Quantum Chemistry Approach on Spectral Densities of BChl a in Solution and in FMO Proteins. J. Phys. Chem. B 2015, 119, 9995-10004.

(48) Scholes, G. D.; Curutchet, C.; Mennucci, B.; Cammi, R.; J.Tomasi, How Solvent Controls Electronic Energy Transfer and Light Harvesting. J. Phys. Chem. B 2007, 111, $13253-13265$.

(49) Mennucci, B.; Curutchet, C. The Role of the Environment in Electronic Energy Transfer: A Molecular Modeling Perspective. Phys. Chem. Chem. Phys. 2011, 13, 1153811550.

(50) Madjet, M. E.; Abdurahman, A.; Renger, T. Intermolecular Coulomb Couplings from Ab Initio Electrostatic Potentials: Application to Optical Transitions of Strongly Coupled Pigments in Photosynthetic Antennae and Reaction Centers. J. Phys. Chem. B 2006, 110, 17268-81.

(51) Kubař, T.; Kleinekathöfer, U.; Elstner, M. Solvent Fluctuations Drive the Hole Transfer in DNA: A Mixed Quantum-Classical Study. J. Phys. Chem. B 2009, 113, 13107-13117.

(52) Dijkstra, A. G.; Tanimura, Y. Correlated Fluctuations in the Exciton Dynamics and Spectroscopy of DNA. New J. Phys. 2010, 12, 055005. 
(53) Olbrich, C.; Strümpfer, J.; Schulten, K.; Kleinekathöfer, U. Quest for Spatially Correlated Fluctuations in the FMO Light-Harvesting Complex. J. Phys. Chem. B 2011, $115,758-764$.

(54) Shim, S.; Rebentrost, P.; Valleau, S.; Aspuru Guzik, A. Atomistic Study of the LongLived Quantum Coherences in the Fenna-Matthew-Olson Complex. Biophys. J. 2012, 102, 649-660.

(55) Wu, J.; Liu, F.; Shen, Y.; Cao, J.; Silbey, R. J. Efficient Energy Transfer in Lightharvesting Systems, I: Optimal Temperature, Reorganization Energy and Spatialtemporal Correlations. New J. Phys. 2010, 12, 105012.

(56) Wu, J.; Liu, F.; Ma, J.; Silbey, R. J.; Cao, J. Efficient Energy Transfer in LightHarvesting Systems: Quantum-Classical Comparison, Flux Network, and Robustness Analysis. J. Chem. Phys. 2012, 137, 174111. 
Graphical TOC Entry

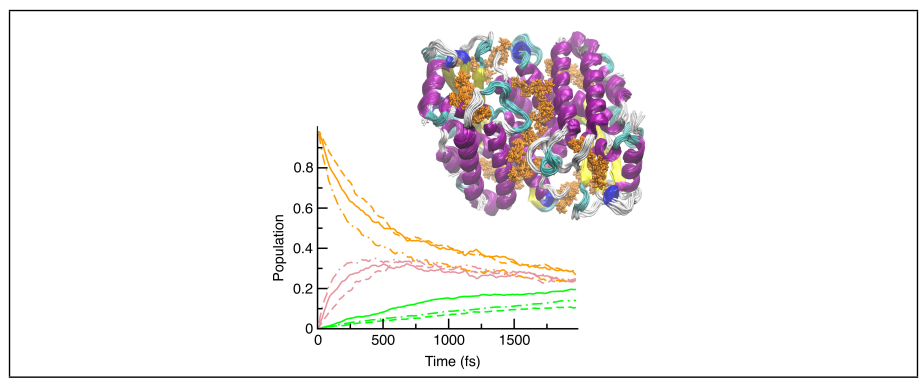

